\title{
A Real-Time IVR Platform for Community Radio
}

\author{
Konstantinos Kazakos ${ }^{1}$, Siddhartha Asthana ${ }^{2}$, Madeline Balaam ${ }^{1}$, Mona Duggal ${ }^{3}$, Amey Holden$^{1}$, \\ Limalemla Jamir ${ }^{3}$, Nanda Kishore Kannuri ${ }^{4}$, Saurabh Kumar ${ }^{3}$, Amarendar Reddy Mamindla ${ }^{4}$, \\ Subhashini Arcot Manikam ${ }^{4}$, GVS Murthy ${ }^{4}$, Papreen Nahar ${ }^{5}$, Peter Phillimore ${ }^{5}$, Shreyaswi Sathyanath ${ }^{6}$, \\ Pushpendra Singh ${ }^{2}$, Meenu Singh ${ }^{3}$, Pete Wright ${ }^{1}$, Deepika Yadav ${ }^{2}$, Patrick Olivier $^{1}$ \\ ${ }^{1}$ Open Lab, Newcastle University, Newcastle upon Tyne, United Kingdom \{firstname.lastname\}@ncl.ac.uk; \\ ${ }^{2}$ Indraprastha Institute of Information Technology, Delhi, India; siddharthaa; psingh; deepikay@iiitd.ac.in \\ ${ }^{3}$ PGIMER, Chandigarh, India \\ ${ }^{4}$ IIPH, Hyderabad, India \\ ${ }^{5}$ Newcastle University, Newcastle upon Tyne, United Kingdom \\ ${ }^{6}$ A J Institute of Medical Sciences, Mangalore, Karnataka, India
}

\begin{abstract}
Interactive Voice Response (IVR) platforms have been widely deployed in resource-limited settings. These systems tend to afford asynchronous push interactions, and within the context of health, provide medication reminders, descriptions of symptoms and tips on self-management. Here, we present the development of an IVR system for resource-limited settings that enables real-time, synchronous interaction. Inspired by community radio, and calls for health systems that are truly local, we developed 'Sehat ki Vaani'. Sehat ki Vaani is a real-time IVR platform that enables hosting and participation in radio chat shows on community-led topics. We deployed Sehat ki Vaani with two communities in North India on topics related to the management of Type 2 diabetes and maternal health. Our deployments highlight the potential for synchronous IVR systems to offer community connection and localised sharing of experience, while also highlighting the complexity of producing, hosting and participating in radio shows in real time through IVR. We discuss the relative strengths and weaknesses of synchronous IVR systems, and highlight lessons learnt for interaction design in this area.
\end{abstract}

\section{Author Keywords}

HCI4D; ICTD; IVR; community; mHealth; resourcelimited settings; India; design; user experience.

\section{ACM Classification Keywords}

H.5.3. Group and Organization Interfaces: Synchronous interaction

Copyright is held by the owner/author(s).

CHI'16, May 07-12, 2016, San Jose, CA, USA

ACM 978-1-4503-3362-7/16/05.

http://dx.doi.org/10.1145/2858036.2858585

(c) (1)

This work is licensed under a Creative Commons Attribution International 4.0 License.

\section{INTRODUCTION}

Web 2.0 has provided unique opportunities for individuals to better manage their health by engaging with peers to form health communities [3,9]. In resource-limited settings, where there are low levels of internet connectivity and numerous challenges due to socioeconomic circumstances (e.g. lack of skills, low levels of literacy), the use of Web 2.0 tools for health management is rarely an option [6,19]. To address this, prior research has highlighted the potential of voice-based platforms to foster collaboration and knowledge exchange between community members for a wide range of different purposes, including health $[12,20,26-28,30,32,33]$.

These systems offer asynchronous communication, which within the context of healthcare allow for interactions such as the pushing of medication reminders, or tips about how self manage a condition [12,27-28,30]. Inspired by the popularity of radio shows in resource-limited settings, and in particular India $[13,34,35]$, as well as calls within the HCI community to explore digital health systems that allow for the local production and sharing of health content [9], we designed, developed and deployed Sehat ki Vaani ('HealthVoice' in English). Sehat ki Vaani is an interactive radio IVR platform that enables community members to create and host radio chat shows (radio hosts) through an Android application, as well as participate in radio shows (as listeners) through their own mobile phones (usually feature phones) without incurring any financial cost. We position this work in relation to $[14,15,34,35]$, which have investigated the role of community radio shows as an engagement medium. We build on this by addressing the technical infrastructure required, as well as the challenges of limited Internet connectivity and scalability.

To understand Sehat Ki Vaani's user experience we conducted a field trial in North India with two local community health workers who as radio hosts prepared and conducted six shows in total on health topics of local relevance using our platform. All in all, 33 listeners tuned in and participated in discussions on Type 2 diabetes and 
maternal health. Our findings highlight the potential for synchronous IVR systems to offer community connection and localised sharing of experience, while also highlighting the complexity of producing, hosting and participating in radio shows in real time through IVR. The contribution of this paper is threefold. Firstly, we develop a novel technological approach that promotes real-time community health discussions in resource-limited settings. Secondly, we generate an understanding of how this approach can be implemented in such settings through the deployment of Sehat ki Vaani in North India. Thirdly, we provide a set of lessons learnt regarding interaction design for IVR systems as tools for synchronous communication.

\section{RELATED WORK}

\section{Asynchronous Voice Platforms in Developing Regions}

Despite the continued roll-out of mobile Internet in developing regions, there exist numerous challenges with its use that are more apparent in resource-limited settings [24] - settings where internet access is intermittent due to technical issues as well as the financial and personal circumstances of the population. Prior research has described and explored the significance and popularity of voice-based systems in enabling individuals within developing regions to communicate, seek information and participate in community discussions $[7,33]$. Such systems utilize Interactive Voice Response (IVR), asynchronous voice platforms that provide the ability to play and record prompts as well as gather phone key input [30].

Previous studies have investigated the role that IVR-based platforms have played within different settings in India. These include embedding answering systems through IVR in community radio stations [15,34], sharing knowledge around farming practices through interactive voice forums [24], motivating citizens to be aware and engage more with their local communities through citizen journalism $[5,20,32]$ as well as giving the opportunity to local community members to exchange ideas regarding employment opportunities [33]. Other studies have explored the use of IVR systems in content creation and dissemination in rural areas and documented the merit of locally created content [1]. The common thread among all these studies is that they support asynchronous interaction between the listeners. Ongoing challenges with these systems exist in the form of poor user experiences manifested in tedious and drawn-out interactions with extensive menu structures [5,18].

\section{From Mobile Text to IVR Systems for Community Health}

Prior research in resource-limited settings has examined the role of mobile technology in supporting self-management of Type 2 diabetes and maternal health in the individual $[10,11,19]$. Other studies have realized more communityoriented approaches towards the management of Type 2 diabetes and maternal health [16,31]. Olmen et al. [23], who studied the effectiveness of text messaging as a Type 2 diabetes self-management education tool in three countries, identified the value of text messages as a means of enabling community members who experience similar challenges to share their experiences. Additionally, Perrier et al. [26] showed how pregnant women in Kenya gained insight into the management of their pregnancy by communicating with a qualified nurse using an automated SMS engagement mechanism. Maitra and Kuntagod [17] designed and deployed a mobile platform specifically crafted to assist maternal health workers in rural India to successfully disseminate pregnancy and child-care related advice. Finally, [2] notes the usefulness of engaging midwives in the design of systems for maternal health.

While the value of text-based health communication and experience exchange has been widely demonstrated, the high levels of illiteracy that exist in resource-limited settings [19] call for alternative (non-text) approaches such as IVR. Within the health context, Joshi et al. [12] highlighted the importance of IVR platforms in assisting individuals with HIV/AIDS who live in resource-limited settings to both access information relating to their illness and sustain direct links with their physician. Furthermore, Rudy et al. [29] implemented and deployed an IVR intervention for melanoma patients who live in rural areas. Their findings highlighted the value of social support through phone discussions among groups of patients. In the context of chronic illnesses, Piette [27] studied the use of IVR systems in facilitating individuals with chronic illness (including diabetes) to support each other through the difficulties and hardships of managing their illness. They underlined the positive effect that the use of an IVR system had for the participants - particularly for those who did not participate in sharing information before the study. Piette et al. [28] used automated and pre-recorded calls on topics surrounding illness management with members of a community in Honduras, and demonstrated the feasibility and high acceptability of this IVR approach in a rural setting.

\section{From Asynchronous to Synchronous IVR Systems for Community Healthcare}

There has been a wealth of research efforts that investigate the role of IVR systems in resource-limited settings. The majority of this work has concentrated on the asynchronous exchange of voice messages between community members. A significant gap exists in the exploration of IVR systems that exploit synchronous low-cost community-led health in resource-limited settings. In response, we designed and developed Sehat ki Vaani - a synchronous IVR platform that is inspired by the popularity of radio talk shows in India $[13,34,35]$. We provide in situ understandings of this approach to assisting individuals in rural India in better managing their health condition through community-led synchronous discussions.

\section{CO-DESIGNING AND DEVELOPING 'SEHAT KI VAANI'}

At the outset of the project we ran a series of design workshops to investigate how mobile technologies could support self-management of chronic conditions in three 
rural Indian communities. 37 individuals participated in these workshops, which involved role-play and the use of low-fidelity prototyping materials to explore experiences, needs and values related to self-management. These workshops highlighted a number of key requirements and interactions for digital technologies that seek to support self-management of chronic conditions in resource-limited settings. First, we learnt that individuals valued connecting with others who are living with the same condition. Participants considered that this would help to foster the sharing of experiences as well as contextualized hints and tips about how to best self-manage a condition within a particular community. Given the focus on health, trust was highlighted as important, with individuals needing to feel that the information shared was legitimate, and that those who were receiving potentially sensitive information could also be trusted. Reflecting findings elsewhere [21,22] our participants highlighted the importance of both the Accredited Social Health Activist (ASHA) and the Auxiliary Nurse Midwife (ANM) as individuals who are highly trusted by, acceptable and accessible to the community. Additionally, the participants highlighted that it was essential any system be free at the point of access, and that it provide support to illiterate service users to access information and voice their opinion on an equal footing.

Given this design space we began exploring concepts related to community-led radio chat shows. We were intrigued as to whether these kind of familiar interactions could be repurposed to enable peer support within communities around issues such as health and selfmanagement. In recognizing the key role of ANMs and ASHAs within community healthcare in rural India, we sought to create a technology design that would enable these key community members to plan and host radio chat shows based on their understanding of a community's particular needs. We saw distinct advantages in utilizing an Interactive Voice Response (IVR) system as the backbone to our prototype, since these systems have proved to be both popular and acceptable to rural Indian users.

Following an iterative, user-centered design cycle, we explored the possible interactions, experiences and acceptability of Sehat ki Vaani through a medium-fidelity prototype. The medium-fidelity prototype was developed using a commercially available VoIP app that enables unstructured group chats using (registered) mobile phones. We returned to the communities who had participated in the earlier design workshops and asked participants to work in small groups to enact mock radio shows using the VoIP app's group-chat facility. Group chats were led by ASHAs, ANMs and a medical practitioner, and the concept was well-received by participants. Contrary to our initial concerns, that listeners would be unwilling to share their experiences with others using this medium, many participants were willing to ask questions and voice their opinions. In fact, the overall experience was for much of the time chaotic, with many individuals trying to talk at once, and at times hold separate conversations with others on the call. We also experienced first-hand the technical challenges entailed in deploying a prototype that relies on internet access within rural regions of India. These initial investigations provided us with a clearer understanding of the technical difficulties we would face when deploying the concept. In addition, we learnt that it would be crucial for hosts to be able to control the structure and content of the show through simple interactions at an interface, i.e. be able to mute and unmute callers, be informed of how many callers were on the line, and keep track of time on air.

\section{Envisioning Sehat ki Vaani}

Sehat ki Vaani is an IVR system that enables individuals to plan a radio chat show, create and distribute trailers for the show, and host an interactive real-time show. It uses existing GSM channels to connect listeners on cheap lowend mobiles with an individual who acts as the radio host through the dedicated Android application. As such, it enables individuals in different settings (including resourcelimited ones) to actively participate in a discussion about key community issues through their own mobile phone. In what follows we present a scenario envisioning the use of Sehat ki Vaani from the perspective of a new listener.

Shettal has recently been diagnosed with Type 2 diabetes, she is fearful that Type 2 diabetes is a life-long sentence and that she will no longer be able to visit her extended family and friends because they won't be able to serve food that is suitable for a diabetic. Her local ASHA has met with Shettal and has recommended that Shettal call-in to a local Sehat ki Vaani diabetes support group. Shettal is anxious at first, but when her ASHA reassures her that it is free to join and all she need do is listen to a show on her mobile she is willing to give it a try. Using her phone, the ASHA registers Shettal's mobile number to receive calls for the local diabetes group, and pushes the most recent trailer to Shettal's phone. Soon after, Shettal receives a call to her mobile phone. On answering, Shettal hears her ASHA describing a show that will be run the following Thursday the show is about cooking for diabetes - Shettal presses ' 1 ' on her mobile phone to register her interest in listening.

When the show starts on Thursday, Shettal receives a call to her mobile phone. Shettal hears the ASHA offers advice about the local seasonal foods she could eat at the moment, and a reminder that she should avoid eating rice. The ASHA tells listeners that to ask a question or share an experience they should press ' 1 '. The ASHA then introduces a local doctor with many years' experience. She describes the importance of having regular eye-tests and other tests to monitor the overall health of a diabetic. The ASHA then introduces a caller, who describes a recipe they have been using to make chapattis healthier. As the show unfolds the ASHA and the local doctor work together to respond to the community's questions. As Shettal listens, and hears how members of the community she lives in manage their diabetes on a daily basis she feels less 
anxious, she even learns how to reduce the oil used in meals she cook's for her family.

\section{Implementing Sehat ki Vaani}

Sehat ki Vaani is based on a client-server architecture and has two different types of clients: Radiohost and Listeners. The Radiohost client is an Android application that runs on an Android-based smartphone or tablet and requires Internet connectivity. The aim of this application is to manage the show and community members' participation in the show. The Radiohost client allows the host to: (i) broadcast a radio show; (ii) stop an ongoing radio show; (iii) monitor the duration of a radio show; (iv) view the number of listeners; and (v) display the list of listeners who want to express their views or have a query for the guest of current show. Furthermore, a host can: (vi) unmute a listener and allow them to speak; and (vii) mute a listener who has finished speaking.

The back-end of the platform consists of a server that is hosted on a cloud-based service (Amazon EC2) and accepts requests from the Radiohost client. The back-end is built on top of a number of open source technologies, including: FreeSWITCH, MySQL, and APACHE Web Server running on a Linux (Ubintu) server. The flow of information and data is moderated through PHP. FreeSWITCH, which is an open source VoIP server, manages all the telephony side of the platform. It accepts audio from the Radiohost client and sends it to all the Listeners (who use low-end mobile phones) using a GSM Service (Doorvani.com). The Apache web server hosts a PHP-based application that accepts the web request from the Radiohost client and updates the relevant table in the MySQL database. FreeSWITCH constantly monitors the relevant tables in the database to perform any requested telephony operation (e.g. dial a telephone number, unmute/mute a caller, etc.), FreeSWITCH updates the database after performing each task that is read by the Radiohost client using a web request submitted to a PHP application.

The platform is completely free for listeners to use. Less than 5 INR per minute was spent running a show. The average duration of a show was around 104 call minutes (sum of all call minutes of all the participants of a show) and we paid 445 INR on average for each show to our VoIP service provider. Therefore, the cost/minute was $445 / 104=$ 4.28 INR.

\section{DEPLOYING SEHAT KI VAANI}

To test 'Sehat ki Vaani' and explore its potential as a technology to supports self-management of health in resource-limited settings we undertook a two-stage deployment in North India. Through an initial pilot we refined the technical infrastructure. This was followed by six radio shows that were planned and hosted in two separate communities in two different settings. Working with local healthcare providers, the radio shows were focused on Type 2 diabetes and maternal health. These topics are of particular relevance in India [25] and the local healthcare researchers within our team considered there to be communities within these regions who would benefit from these foci, and would therefore have an interest in participating.

\section{Location of Pilot and Deployment}

We piloted and then deployed Sehat ki Vaani in two locations in North India. Our first site was Chandigarh (450km northeast of Delhi). Chandigarh has a population of over 2 million. For our deployment we were situated in a district within the outskirts of the city, where the local population was served by the dispensary clinic. Our second site was Naraingarh, $55 \mathrm{~km}$ east of the Chandigarh. Naraingarh has a population of 30,000 and the medical needs of the population are served by a local hospital.

\section{Piloting Sehat ki Vaani}

We spent one-week piloting Sehat ki Vaani on location. Four members of the research team acted as listeners, with one further member acting as the guest. We recruited two Community Health Workers (one ANM and one ASHA) to act as radio hosts, both throughout the pilot and in the final study. Throughout the pilot we ran multiple shows, testing and refining in each case the capabilities of the system. This included: the ability to register individuals that are interested in listening to the show (in this case team members); the ability to create and distribute a trailer for the show to registered numbers; the ability to initiate a radio show from the Radiohost client and call all registered listeners; the ability for a caller to register their interest in contributing to the show through dialing 1 ; and the ability to mute and unmute listeners who have dialed 1 during the show.

Piloting the system, allowed us to better understand the situation on the ground. We discovered that the Radiohost client made numerous unnecessary pull requests to the IVR server, which overloaded the connection. We reengineered the system so that the IVR sever would push information to the Radiohost client only when necessary, significantly reducing the network-usage of the application, and increasing the system's stability. The telephony networks were less stable than we originally anticipated, which resulted in difficulties in connecting to some participants. To address this we added a new error control mechanism to the back-end, and extended the number of retries to 20 to improve the likelihood that listeners received a phone call from the system.

\section{Participant Information}

We recruited three types of participants - radio hosts, guests and listeners - who were all given a mobile airtime gift card in exchange for participation in the study.

\section{Radio Hosts}

We invited the local ANM and ASHA to be the radio hosts. The radio host in Chandigarh was an ANM (45 years old) who had almost 20 years of experience. She was responsible for approximately 1,000 residents who all resided in the vicinity of the dispensary clinic. She had a 
good understanding of Type 2 diabetes management as well as being well known to the community. The ANM spoke fluent Hindi and was a competent English speaker. She had little experience of using digital technologies beyond her mobile phone. The Naraingarh radio host was an ASHA (38 years old) who had almost five years of experience. She was also well known in her community and was responsible for 200 individuals (of a total population of 3,000). The ASHA spoke fluent Hindi, only a little English, and, similar to the ANM, had little experience of using digital technologies beyond her mobile phone.

\section{Guests}

A female clinician, who was a member of the research team, acted as the guest for each of the radio shows (referred to using the pseudonym Dr. Savi throughout the analysis). She had extensive knowledge of both Type 2 diabetes and maternal health. She was proficient in interacting with Android based applications on both tablets and smartphones. Finally, she had a good pre-existing relationship with both the ANM and the ASHA.

\section{Listeners}

The ANM and the ASHA invited individuals from their communities to participate. In total, thirty-one participants $(\mathrm{N}=31)$ showed interest in listening to, and participating in, the radio shows. Table 1 characterizes our listeners.

\begin{tabular}{c|cc} 
Listeners & Chandigarh & Naraingarh \\
\hline $\begin{array}{c}\text { Health Topic } \\
\text { Registered }\end{array}$ & Diabetes & Pregnancy \\
Listeners & $N=11$ & $N=20$ \\
Mean Age & $50 \pm 12.9$ & $25 \pm 2.7$ \\
Sex (M, F) & $7 M, 5 F$ & $18 F, 1 M$
\end{tabular}

Table 1. Listeners (by study location).

Listeners came from diverse socio-economic backgrounds and did not own a smartphone. Most of our listeners were using mobile devices that were accessed by other family members. 28 of the 31 listeners considered themselves inexperienced users of mobile phones.

\section{Data Collection}

We utilized questionnaires, semi-structured interviews and observations to evaluate Sehat ki Vaani. Before the deployment we asked listeners to provide demographic information through a simple survey (see Table 1). Throughout the study we conducted, where possible, informal interviews with listeners who had dialed ' 1 ' to participate in the show (these listeners are referred to as SL 'Speaking Listeners' in Table 2.) These interviews were not audio recorded, but notes were made and collated as part of each show debrief. Finally, we interviewed the radio hosts after the final shows at each location. The interviews and shows were audio recorded, translated, and transcribed. Observational field notes were written every evening after the running of a show, to maintain a record of the atmosphere and moment-by-moment workings of each show. In total, we collected interviews with 11 listeners, two exit interviews with the radio hosts, ten pages of observations, 26 demographic questionnaires, and six audio recordings of the shows. Log data was collected for the IVR server to document the duration of each radio show, the number of individuals who were registered as interested in listening to a show (Registered Listeners), the number of listeners who chose to accept the call from the IVR server and listen to a show (Actual Listeners), as well as the number of listeners who dialed 1 to participate in a show (Speaking Listeners).

\section{Data Analysis}

The data collected from our deployment was analyzed using an inductive thematic analysis approach [4]. Once the study was completed and the material was gathered, five of the team members (four Indian and one from the UK) listened to each recorded show and went through the related interview data. While listening to the shows, the Hindispeaking team members translated parts of the show, which were then discussed amongst all team members in English. In our analysis we were interested in the hosts' and the listeners' experiences, the content shared and the obstacles encountered during the deployment. The codes were then analyzed and themes emerged. This iterative process resulted in a set of themes that described the experience of use as it unfolded.

\section{FINDINGS}

In this section we present a brief summary of the radio shows produced during the deployment. We then describe the role that confidence played in a show's production, the work entailed in producing shows and the experience of listening and participating.

\section{Summary of Radio Shows}

All shows were run in Hindi. Table 2 presents a summary of these shows. In both locations, and across both topics, the radio shows themselves had similar numbers of Actual Listeners (AL), and similar numbers of Speaking Listeners (SL, those who pressed ' 1 ' and spoke during the shows).

\begin{tabular}{|l|c|c|c|c|c|c|}
\hline & \multicolumn{3}{|c|}{$\begin{array}{c}\text { Chandigarh } \\
\text { Type 2 Diabetes }\end{array}$} & \multicolumn{3}{c|}{$\begin{array}{c}\text { Naraingarh } \\
\text { Maternal Health }\end{array}$} \\
\hline Show & $\mathbf{1}$ & $\mathbf{2}$ & $\mathbf{3}$ & $\mathbf{1}$ & $\mathbf{2}$ & $\mathbf{3}$ \\
\hline Topic & Diet & $\begin{array}{l}\text { Diagnosis/ } \\
\text { Treatment }\end{array}$ & $\begin{array}{c}\text { Peer } \\
\text { Support }\end{array}$ & $\begin{array}{c}\text { During } \\
\text { Pregnancy }\end{array}$ & $\begin{array}{c}\text { During } \\
\text { Delivery }\end{array}$ & $\begin{array}{c}\text { After } \\
\text { Birth }\end{array}$ \\
\hline DUR & 23 & 22 & 26 & 32 & 23 & 48 \\
\hline RL & 11 & 11 & 11 & 20 & 20 & 20 \\
\hline AL & 5 & 4 & 6 & 4 & 6 & 8 \\
\hline SL & 3 & 3 & 5 & 4 & 3 & 6 \\
\hline RpL & - & 4 & 4 & - & 3 & 3 \\
\hline
\end{tabular}

Table 2. Details of each radio show in each location, including the duration in minutes (DUR), the number of Registered

Listeners (RL), the number of Actual Listeners (AL), the number of listeners who dialed ' 1 ' to speak on the show (SL), and the number of Repeat Listeners (RpL).

Across the three shows in both locations we saw a slight increase in the number of Registered Listeners who actually 
chose to listen to the show through accepting the call from system. We also notes an upward trend in the number of Speaking Listeners, those who chose to dial ' 1 ' and verbally participate in the show through asking a question, or sharing their experience. The differences in radio show duration between Naraingarh (average $=34$ minutes) and Chandigarh (average $=23$ minutes) were in part a result of technical difficulties experienced deploying Sehat ki Vaani in Naraingarh. During shows hosted in Naraingarh there were a number of occasions where the callers and the host were unable to hear one another. This was the result of lower speed Internet connection available to us in Naraingarh, which influenced the streaming quality of the voice, as well as relatively poor local telephony network, which meant listeners frequently lost telephone reception during the shows.

\section{Confidence in Hosting, Talking and Informing}

In asking our hosts to run three radio shows about health conditions we were undoubtedly asking our volunteer ANM and ASHA to do something they had no experience doing previously. Both hosts faced this challenge with some trepidation.

'When we do our first show, as everybody knows there are a few inhibitions before you do a new job. How will it be? How will it be done? How will we talk to so many people at the same time? How we'll be able to handle a group of 10-15 people? So there was a little fear in our hearts. But we had become pretty confident by the time we conducted our third show.' (ANM Host, Chandigarh)

The ANM and ASHA were quite understandably worried about whether they would be able to run a chat-show live, and how the prototype platform we had developed would enable them to achieve this.

In preparation for each show the hosts spent quite some time working with their guest (who was a member of the research team with medical training) scripting closely an introductory segment for the show which would both inform the listeners of the show's particular topic, as well as provide some useful information to help the listener manage their diabetes, or pregnancy. In each case, as the show went live, the host would read their prepared paper script verbatim, often holding and fidgeting with the script throughout.

Despite having concerns about how the show was going to go and being anxious about being listened to by a number of people connected through the system, our hosts provided clear information for their listeners, conveying their messages often with confidence and flair. For example, in the introductory segment for the first diabetes show, the ANM host starts the show by offering the following concrete advice:

"Like I told you earlier, that today we will emphasize on dietary habits and physical exercise, so please pay attention to what I am about to speak. [...] Now you must reduce your rice and potato intake, as for chapattis, you can take wheat chapattis, and also mix besan flour or soyabean flour to it. You can also eat Millet or
Tide chapattis. And what about fruits? All the fruits you are having should be fresh fruits. You should limit your intake of sweet fruits that are too sweet like banana, grapes, mangoes, chikoo etc."

(ANM Host, Chandigarh, Show 1)

Despite the work entailed in planning the shows, registering listeners, and the somewhat nerve-wracking experience of running the shows, both the ASHA and ANM described the activity as being worthwhile:

"[...] we could also give our message to all of them at the same time, unlike in clinics where we have to repeat the same stuff again and again like take care of your diet, do some physical exercises, take you medicine regularly. One has to keep repeating everything. If 50 patients visit us every day we have to repeat everything 50 times."

(ANM Host, Chandigarh)

As the hosts gained more experience of both hosting shows, and using the prototype platform, they exhibited more confidence in their approach to introducing shows. For example, during the debrief at the end of the second diabetes show, the ANM decided to make her introductory scripts shorter and to use more informal and persuasive language to make the overall introduction more attractive to her listeners.

Initially, there was concern that no listeners would opt to dial into the show, However, from the first show onwards we found that members of the community were willing to dial into the show, particularly to ask medical questions of the hosts. Listening to the contributions made by listeners, it is clear that the platform requires listeners to be confident and motivated to ask questions, and to share their own insights with an (unknown) community of listeners. In the following a male caller joins the radio show, and the ANM host coaxes the listener to describe elements of his diabetes self-management, despite the caller considering that no one will pay attention to him as a consequence of his ethnicity.

Host ANM Chandigarh - "Ok, no, no, when we started the show, did you hear the gentleman speak on the show, when we started discussing about the frequency of blood tests, did you follow it? Whatever he spoke."

Listener Male 1 - "Yes, Yes, I understood, he was right, there was nothing incorrect in what he said, whatever he said about blood sugar was right."

Host ANM Chandigarh - "What did you understand? Will you speak something on it, so that others can also listen to you."

Listener Male 1 - "Well actually whatever he said was correct, but since I'm a Bengali, whatever I will say no one will able to follow, yes please."

Host ANM Chandigarh - "Brother, everyone will follow you, don't worry, just speak up, ok."

Listener Male 1 - “...I am telling something else...I keep taking my medicine, in the afternoon and at night..."

(Chandigarh, Show 2)

\section{Keeping the Show on the Road}

For a successful show the host needs to be able to keep the show on the road in the face of unexpected events and 
complications. This includes encouraging listeners to call in to offer their advice, experiences, or to ask questions, as well as filling in for what the research team began to refer to as 'black holes'. These were points within the show where there would be periods of silence. These 'black holes' typically occurred when listeners did not opt to ask questions after the host had introduced the show, when the host was not sure how to respond to listeners' questions, and when there were significant technical difficulties.

The radio hosts in both locations found it extremely difficult, particularly during the first shows, to fill these 'black holes' while they waited for a listener to be brave enough to dial ' 1 '. For example, during the first show, after the Chandigarh host had completed her introduction, there were no callers on the lines. She repeated six times "Hello press 1 to speak" but she received no reply. On this occasion, the ANM stopped talking and turned her gaze to the guest (and member of our research team) who was sitting next to her. Both were at a loss of what to do, or what to say, uncertain whether anyone was listening. After approximately 90 seconds of complete silence the ANM introduced the guest to initiate a discussion of her views and experiences related to diet and exercise in the selfmanagement of diabetes. As the shows progressed both of the hosts exhibited increased skill in managing periods where no callers were waiting with questions or comments. In the following excerpt, the ANM in Chandigarh has finished a conversation with a previous caller. On realizing that no listeners were waiting to speak, the ANM first prompted listeners to dial 1 , in order to have their say, and then immediately stuck up a conversation with the host:

"Thank you so much...other participants who are connected to us, and if they can hear my voice I once again request them to dial no 1 from your phone. So that we can talk to rest of the people like we have talked to people earlier, and people are also benefited with this conversation. So we have Dr. Savi with us, and she is our guest...so she will tell you something about blood sugar. So yes Dr. Savi thank you so much for joining us on our show. Dr. Savi today that we have made a role of peer support, one sugar patient can talk to other, how will it mutually help them? So please enlighten everybody regarding this so that people can take advantage." (ANM Host Chandigarh, Show 2)

Both hosts displayed increasing skill at encouraging listeners to take part in their shows. In Chandigarh the host began to refer to listeners as 'friends' in a bid to make participating feel less threatening and formal.

\footnotetext{
"It was great talking to you, but please stay on line, please don't cut the phone, because we will speak to our other friends too, and you can also listen to them. Thank you so much..."
}

(ANM Host Chandigarh, Show 3)

'Black holes' and periods of confusion also emerged when hosts unmuted callers so they could join the show. These 'black holes' were caused by the time-lag between the host interacting with the Radiohost client (i.e. selecting to unmute a caller), the application updating the back-end, and the listener realizing they could speak.
Host ANM Chandigarh - "Yes, no.3,7,3 can you hear us? Yes, madam?... no. 3,7,3 can you hear us?..."

Listener Female 2 - "Yes madam, hello, hello. Yes madam."

Host ANM Chandigarh - "Can you hear us? Yes the signal is somewhat weak but I can hear you...so now you can hear us clearly."

Listener Female 2 - "Right, yes madam. Now I can hear you, all right."

Host ANM Chandigarh - "Ok now we have had some discussion which you must have heard. Now will you please tell us that how do you manage your diabetes? People who have joined us and are listening to us right now will also hear you, and the way you manage your diabetes...madam."

(Chandigarh, Show 3)

Hosts also found it challenging to manage the listeners who called in. In practice, the host had to achieve four connected goals: 1) to encourage the caller to talk; 2) to help the caller make the point that they phoned in with; 3) to address the questions and topics of conversation the caller wanted to raise; and, 4) to ensure callers do not outstay their welcome. One strategy utilized by the ANM was to begin their conversation with a new caller by questioning that caller in relation to previous content. This strategy appeared to have the dual effect of making new callers feel comfortable and confident talking, as well as ensuring a coherent narrative to the show:

Host ANM Chandigarh - "Did you hear what we discussed about sugar today?"

Listener Male 1 - "Yes, yes that lady...that girl was talking to you just now...she was saying something about sweeping floor."

Host ANM Chandigarh - "Yes, Yes."

Listener Male 1 - "Yes, Yes, Yes I heard they whole conversation, So the no... well its ok as far as I am concerned, I go for long walks and all, ok, it's ok ... so what about eating habits, yes please speak."

(Chandigarh, Show 2)

The hosts were often uncertain as to what to do if a listener began moving off topic, or spoke for too long. In general, the hosts would wait until the caller had finished their question or completed sharing their opinion before proceeding to the next caller, regardless of whether the points raised continued to be on topic. For example, during the third show on maternal health in Naraingarh, which was on the topic of breastfeeding and infant feeding, the ASHA struggled to initiate conversations with listeners about breastfeeding, with callers preferring to discuss their immediate problems (listeners were pre-partum).

Host ASHA Naraingarh - "So in our show today we...like you breastfeed or otherwise feed your child...you know about it all."

Listener Female 1 - "Well yes."

Host ASHA Naraingarh - "Ok so now... as you deliver the baby ...the moment you start lactating ...immediately after the delivery...you have to definitely feed your child with that thick yellow milk..."

Listener Female 1 - "Ok Madam.” 
Host ASHA Naraingarh - "It is very important for the baby."

Listener Female 1 - "Yes ok."

Host ASHA Naraingarh - "Don't take it out and waste it, feed it to the baby."

Listener Female 1 - "Ok madam. Right."

Host ASHA Naraingarh - "Do you want to ask anymore questions."

Listener Female 1 - "No Madam. everything else is alright, only I have difficulty in breathing."

Host ASHA Naraingarh - "Oh not to worry, it is because of the hemoglobin deficiency in your body that you have trouble breathing. OK. So not to worry, take fenugreek and well protein rich diet like pulses. So when you take these things in routine, calcium and iron levels in your body will automatically start increasing. Your need not worry."

Listener Female 1 - "Madam this one thing that I wanted to ask was, about the trouble in breathing, I feel so suffocated."

Host ASHA Naraingarh - "Ok. Dr. Savi is with us, she will talk to you, you can ask her about this and what should be done about it?... Ok, so Dr. Savi has joined us ...you can talk to her..."

(Naraingarh, Show 3)

Dr. Savi continued a conversation with the listener for some time, discussing the importance of increasing and monitoring the listener's iron intake to help relieve the feeling of breathlessness before the show was moved back to the topic of breastfeeding by the ASHA.

Even when hosts realized that there was another caller on the line, they found it difficult to finish one conversation and move to the next. For example, on one occasion the radio host in Naraingarh noticed that two listeners were in the queue to speak, but despite the current caller having already spoken for approximately five minutes the host only ended the conversation when a research team member signaled to her to move on.

\section{The Act of Listening and Sharing}

For listeners, the act of listening and sharing during radio shows provided answers to questions and concerns, and provided comfort to listeners through the act of connecting. Nevertheless, this act of listening at times resulted in a frustrating, boring or confusing experience. Similar to our hosts, listeners had to contend with an audio experience that did little to communicate the status of the show in times of technical difficulty. Poor telephony networks and crossed lines meant there were often ghost voices in the background. Changes in reception strength and connectivity meant the volume speakers' voices sometimes drifted in and out of audibility. The limited support for those dialing ' 1 ' and seeking to participate in the show also caused frustration and confusion. Listeners received no indication as to how long they would need to wait before they would be live. For example, during the second show in Chandigarh a listener pressed ' 1 ' at the end of the introduction. He then had to wait 12 minutes before he was invited to participate in the show by the host. By the time the host invited him to share his thoughts, the listener had hung up, reporting in a later informal interview that he had grown tired of waiting, even though during this wait time he had been able to listen the show.

When listeners were able to successfully contribute, there contributions fell into two main categories: sharing experience, and asking direct medical questions. In what follows, one listener describes how he manages his diabetes:

Host ANM Chandigarh - "We were trying to ask you, that have you done any thing else for controlling your blood sugar? So that your blood sugar stays in control. You know people tend to take other measures apart form medicine for controlling their blood sugar."

Listener Male 2 - "Yes I go for long walks, I also make bitter gourd juice at home and drink it on an empty stomach. I get the sugar tests done in a month or two, this ensures me of my controlled blood sugar. I also got my whole body check up done once along with skin test and all. What ever tests were done in laboratory their reports were all normal. The pathologist told me to eat onions, you know, when I eat my food to add onions in my diet."

(Chandigarh, Show 1)

However, the majority of listeners' sought to solicit medical advice. Topics for such questions ranged from modes of storing insulin, through to calcium and iron supplements during pregnancy. At times, listeners shared potentially sensitive personal information, such as the results of blood tests, and the medications doctors had prescribed for them.

Host ANM Chandigarh - "Other participants, if you can hear us, what we have discussed with people here, if others can hear us please dial 1 from your number, so that we are able to talk to you. Number 5, 9, 3.”

Listener Male 2 - "Yes doctor madam please tell me why I become dizzy after waking up early in the morning, what should I do about it."

Host ANM Chandigarh - "Well actually ..."

Listener Male 2 - "Actually sugar, my blood sugar becomes low. So what should I do?"

Host ANM Chandigarh - "We have a guest Dr. Savi with us for answering you question, Dr. Savi will talk to you now."

Listener Male 2 - "Yes, yes."

Female 1 - "So you can discuss your problem with her now, Dr. Savi welcome to the show."

Listener Male 2 - "Yes, yes."

Female 1 - "So Dr. please tell us that what all thing effect diabetes."

Listener Male 2 - "Namaste Dr. Madam."

Dr. Savi - "Namaste. Namaste."

Listener Male 2 - "Dr. Madam I am a resident of Narayangarh."

Dr. Savi - "Ok, alright.” 
Listener Male 2 - "Dr. Madam my question to you is, I feel dizzy in the morning after waking up because my blood sugar level becomes low, what can I do about it?"

Dr. Savi - "Ok, now if your blood sugar is getting low in the morning that means you're not taking correct dose of the medicine prescribed, also whatever medicine you are taking at night, if you take any other medicine along with it, every medicine has its own way of working and they work at different time and in different manner so your low blood sugar levels can be due to this."

(Chandigarh, Show 2)

While the act of sharing and participating required effort and patience on the part of listeners, those who did share and participate in the radio shows declared the benefits to others of doing so:

Host ANM Chandigarh - "Ok. What else would you like to share with your friends."

Listener Female 2 - "Since the day I have connected with your show, I have found great relief, and my heart is also filled with peace and hope that our disease can go away."

Host ANM Chandigarh - "We also feel joyous, when someone says something like that. In fact, it's a great feeling ...well that even if the disease does not completely go away but you are kind of relieved and hopeful that you can get better."

(Chandigarh, Show 3)

And for others, the act of participating in the show enabled them to feel connected and therefore more committed to themselves to continue their efforts at managing their condition:

Listener Female 1 "[...] I am alright now, since I have connected to you, when we connect with someone, and what ever they say ...it kind of sets in your mind. So I feel myself extremely benefitted."

(Chandigarh, Show 3)

\section{DISCUSSION}

This paper contributes to a substantial body of research investigating the role of digital technologies in improving healthcare provision in resource-limited settings. We have explored the possibilities for IVR systems to support realtime synchronous communication within communities, and specifically the running of community-led, health-based radio chat shows. In what follows, we discuss the potential opportunities for synchronous IVR communication and compare and contrast these with those reported for asynchronous systems. We also outline interaction design challenges and lessons learnt for designing for synchronous communication using IVR platforms.

\section{Building a Community or Accessing Information}

In total 33 listeners tuned into six radio shows hosted through 'Sehat ki Vaani', and 24 listeners dialed '1' to participate in the shows. Sehat ki Vaani had been designed to offer communities opportunities to create informal, local peer support networks. We aspired to, as Grimes et al. eloquently state, create a "deeply local and culturally informed health application" [9]. We hoped community discussions would be promoted through the metaphor of a radio chat show, where the structure and content of each show was guided by the local cultural context. However, the majority of users utilized the shows, and the opportunities they created differently. Many of the contributions from listeners focused on seeking formal information about the management of their condition from the radio host, who they considered an expert. There were technical complexities entailed in utilizing an IVR system to enable synchronous communication, as well as interactional and personal challenges faced by ANMs and ASHAs when hosting live shows. As such, we must consider whether an asynchronous IVR system, similar to those detailed in $[12,28]$ might in fact offer a better user experience, enabling users to seek the answers they require, without the sometimes confusing and frustrating experiences entailed with Sehat ki Vaani. Asynchronous IVR systems have been used to provide medication reminders, information about symptoms, and advice about self-management practices and strategies to registered patients. Such information and advice would have very likely been beneficial to the communities we worked with, and particularly those listeners to the maternal health shows, where much of the discussion focused on when and how particular vitamins should be taken. Indeed, our ANM and ASHA hosts both reported that the primary advantage they saw of 'Sehat ki Vaani' was the ability to give the same information to large numbers of patients at the same time, rather than having to repeat advice time and time again. Nevertheless, there are some indications that offering real-time communication through synchronous IVR systems has benefits over traditional IVR. Both [10,29] highlight the relative benefits of real-time peer support, from simple phone calls between similar patients leading to individuals feeling better about themselves [29], through to helping individuals stay healthy, or undertake actions to become healthier [10]. Similarly, in our short deployment we found that listeners spontaneously told others that connecting through the shows offered relief and comfort, and in the case of one listener that this feeling of connection might help him to stay healthy in the future. In the context of resource-limited settings, the capability to create highly local peer-support systems is one distinct benefit of synchronous IVR systems. Indeed we see the potential of Sehat ki Vaani to offer the type of localised health support called for in [9]. Although this wasn't borne out in our short deployment, we saw glimpses of this kind of interaction occurring. For example, through the ANM's description of the fruits and vegetables currently in season that would benefit the diet of a diabetic. Future, deployments of longer durations that explore different configurations of hosts will be necessary to determine if such platforms are feasible, effective and sustainable.

\section{Lessons Learnt for Interaction Design for Synchronous IVR Communication}

Our deployment utilized an extremely simple interface, which provided the host with real-time information on the duration of the show, the number of current listeners, and the number of listeners waiting to speak (who she could 
mute or unmute). However, as evidenced throughout our data, hosting shows like these in real time, with little experience or training, is challenging particularly when taking into account infrastructural issues. Hosts had to manage 'black holes' and fill awkward silences, they had to continually encourage listeners to participate, and when listeners did participate, they had to ensure that those listener contributions were on-topic, as well as find meaningful ways to respond. Listening and contributing to the show brought its own set of complications. In addition to providing more training, we consider that many of these challenges could be overcome through better interaction design of both the IVR system and the Radiohost client.

\section{Support the host in managing listeners}

Taking account low levels of literacy, which might affect the host as well as the listener, we suggest that the Radiohost client make use of color, shape and simple clientside animation to support easy and intuitive use. For example, a circle could be used to represent each listener who has dialled ' 1 ' to participate in the show. This circle could grow in size to indicate the length of time the listener has been waiting, enabling the host to simply and easily prioritise callers. Coupled with this, the IVR system could support the live production of the radio chat show by including a simple and short menu system that a listener navigates when they dial ' 1 '. This interaction would help the listener associate their contribution with a keyword (described by the host before the show). In turn, the Radiohost client could categorise each of the circles representing callers using color to help the host quickly see how to create a show with a coherent narrative. Finally, the Radiohost client should provide real-time feedback to the host regarding the amount of time they have spent with each caller. Here, we suggest that the circle representing the listener currently on the show should blink, with the blink increasing in speed over time to indicate elapsed time. A final color change could indicate when the host really must wrap up the call and move on to another listener.

\section{Prepare listeners for their air-time}

Listeners often joined the show with no warning and with unpredictable delays, leading to confusion as to who could hear whom, and who should speak next. To address this we suggest that the Radiohost client should provide a clear indication to the host when: a) a listener is waiting to join the show; and, b) a connection with a listener has been successfully established (giving the host time to segue between listeners). In addition, we consider that an aural cue - perhaps both a spoken prompt (reminding the listener of the topic of the show and the keyword they selected for their contribution) followed by a set of beeps at increasing speed - should be provided to the listener to communicate to them that a connection with the show is established and they will soon be asked to speak live on the show.

\section{3) Offer support in filling 'black holes'}

The telecommunications infrastructure in many resourcelimited settings does not provide a stable quality of service, and therefore 'black holes' (resulting from one of a number of technology failings) must be addressed. We suggest the Radiohost client offers the host the option to pre-record content that can be integrated into the show at the push of a button. Such content might include adverts for upcoming shows, a spoken description of routes to participation for listeners, music, or even segments of content collected from another host's show (from another location). In addition, the system needs to degrade gracefully at times when the host's connection to the server (and therefore the show) momentarily fails. Here the server should immediately begin playing pre-recorded content while the host reestablishes their connection.

\section{CONCLUSION AND FUTURE WORK}

Sehat ki Vaani is an initial step towards the development of an IVR platform that enables synchronous communication in resource-limited settings, and in this configuration, community-led radio. Our six shows cannot tell the whole story of what is achievable with such a medium, but do point to its potential. The immediate connection offered by synchronous voice-based communication led to feelings of connection and comfort in some of listeners. Our hosts were able to utilize the platform to offer localized support to those in their community in an efficient manner. Both these achievements are indicative of steps taken toward the goal of offering highly local and personalized health services. We continue to develop Sehat ki Vaani and are currently applying our lessons learnt. Three further deployments are planned in a range of settings, including a refugee camp in Lebanon, a rural community in North Yorkshire in the UK, and rural India.

\section{ACKNOWLEDGEMENTS}

This research was supported by an EPSRC award (EP/J00068X/1) and DEITy, Government of India, (through the ITRA project, ITRA/15(57)/Mobile/HumanSense/01). Data supporting this publication is not openly available due to ethical considerations. Access may be possible under appropriate agreement. Additional metadata record at 10.17634/103234-1. Please contact Newcastle Research Data Service at rdm@ncl.ac.uk for further information or access requests.

\section{REFERENCES}

1. Sheetal K. Agarwal, Arun Kumar, Amit Anil Nanavati, and Nitendra Rajput. 2009. Content creation and dissemination by-and-for users in rural areas. Proceedings of the 3rd International Conference on Information and Communication Technologies and Development, IEEE Press, 56-65.

2. Abdullah Al Mahmud and David V. Keyson. 2013. Designing with Midwives: Improving prenatal care in low resource regions. In Proceedings of the 7 th International Conference on Pervasive Computing Technologies for Healthcare, ICST (Institute for Computer Sciences, Social-Informatics and Telecommunications Engineering), 180-183. 
http://doi.org/10.4108/icst.pervasivehealth.2013.25203 2

3. Christophe Boudry. 2015. Web 2.0 applications in medicine: trends and topics in the literature. Medicine 2.0 4, 1: e2. http://doi.org/10.2196/med20.3628

4. Virginia Braun and Victoria Clarke. 2006. Using thematic analysis in psychology. Qualitative Research in Psychology 3, 2: 77-101. http://doi.org/10.1191/1478088706qp063oa

5. Dipanjan Chakraborty and Aaditeshwar Seth. 2015. Building Citizen Engagement into the Implementation of Welfare Schemes in Rural India. In Proceedings of the Seventh International Conference on Information and Communication Technologies and Development, ACM, 22:1-22:10.

http://doi.org/10.1145/2737856.2738027

6. Arul Chib, Michelle Helena van Velthoven, and Josip Car. 2015. mHealth adoption in low-resource environments: A review of the use of mobile healthcare in developing countries. Journal of Health

Communication 20, 1: 4-34. http://doi.org/10.1080/10810730.2013.864735

7. Krittika D'Silva, Meghana Marathe, Aditya Vashistha, Gaetano Borriello, and William Thies. 2014. A Mobile Application for Interactive Voice Forums: Design and Pilot Deployment in Rural India. In Proceedings of the Fifth ACM Symposium on Computing for Development (ACM DEV-5 '14). 121-122.

http://dx.doi.org/10.1145/2674377.2678270

8. Dean Giustini. 2006. How Web 2.0 is changing medicine. BMJ 333, 7582: 1283-1284. http://doi.org/10.1136/bmj.39062.555405.80

9. Andrea Grimes, Martin Bednar, Jay David Bolter, and Rebecca E. Grinter. 2008. EatWell: Sharing Nutritionrelated Memories in a Low-income Community. In Proceedings of the 2008 ACM Conference on Computer Supported Cooperative Work, ACM, 87-96. http://doi.org/10.1145/1460563.1460579

10. Michele Heisler and John D. Piette. 2005. "I help you, and you help me": facilitated telephone peer support among patients with diabetes. The Diabetes Educator 31, 6: 869-879.

http://doi.org/10.1177/0145721705283247

11. Tad Hirsch. 2009. Communities real and imagined: designing a communication system for zimbabwean activists. In Proceedings of the fourth international conference on Communities and technologies (C\&T '09). 71-76.

http://dx.doi.org/10.1145/1556460.1556472
12. Anirudha Joshi, Mandar Rane, Debjani Roy, Nagraj Emmadi, Padma Srinivasan, N. Kumarasamy, Sanjay Pujari, Davidson Solomon, Rashmi Rodrigues, D.G. Saple, Kamalika Sen, Els Veldeman, and Romain Rutten. 2014. Supporting treatment of people living with HIV / AIDS in resource limited settings with IVRs. In Proceedings of the SIGCHI Conference on Human Factors in Computing Systems (CHI '14). 1595-1604. http://dx.doi.org/10.1145/2556288.2557236

13. Zahir Koradia, Piyush Aggarwal, Aaditeshwar Seth, and Gaurav Luthra. 2013. Gurgaon idol: a singing competition over community radio and IVRS. In Proceedings of the 3rd ACM Symposium on Computing for Development (ACM DEV '13), Article 6, 10 pages. http://dx.doi.org/10.1145/2442882.2442890

14. Zahir Koradia, C. Balachandran, Kapil Dadheech, Mayank Shivam, and Aaditeshwar Seth. 2012. Experiences of deploying and commercializing a community radio automation system in India. In Proceedings of the 2nd ACM Symposium on Computing for Development (ACM DEV '12), Article 8, 10 pages. http://dx.doi.org/10.1145/2160601.2160612

15. Zahir Koradia and Aaditeshwar Seth. 2012. PhonePeti: exploring the role of an answering machine system in a community radio station in India. In Proceedings of the Fifth International Conference on Information and Communication Technologies and Development (ICTD'12). 278-288. http://dx.doi.org/10.1145/2160673.2160708

16. Neha Kumar and Richard J. Anderson. 2015. Mobile Phones for Maternal Health in Rural India. In Proceedings of the 33rd Annual ACM Conference on Human Factors in Computing Systems (CHI '15), 427436. http://doi.org/10.1145/2702123.2702258

17. Anutosh Maitra and Nataraj Kuntagod. 2013. A Novel Mobile Application to Assist Maternal Health Workers in Rural India. In Proceedings of the 5th International Workshop on Software Engineering in Health Care, IEEE Press, 75-78.

18. Akhil Mathur and Sharad Jaiswal. 2013. Exploring the interplay between community media and mobile web in developing regions. In Proceedings of the 15th international conference on Human-computer interaction with mobile devices and services (MobileHCI '13). 318-327. http://dx.doi.org/10.1145/2493190.2493201

19. Indrani Medhi, Somani Patnaik, Emma Brunskill, S.N. Nagasena Gautama, William Thies, and Kentaro Toyama. 2011. Designing mobile interfaces for novice and low-literacy users. ACM Trans. Comput.-Hum. Interact. 18, 1, Article 2 (May 2011), 28 pages. http://dx.doi.org/10.1145/1959022.1959024 
20. Preeti Mudliar, Jonathan Donner, and William Thies. 2012. Emergent practices around CGNet Swara, voice forum for citizen journalism in rural India. In Proceedings of the Fifth International Conference on Information and Communication Technologies and Development (ICTD '12). 159-168. http://dx.doi.org/10.1145/2160673.2160695

21. S. L. Norris., F. M. Chowdhury., K. Le Van., T. Horsley., J. N. Brownstein., X. Zhang., L. Jack, and D. W. Satterfield, (2006), Effectiveness of community health workers in the care of persons with diabetes. Diabetic Medicine, 23: 544-556. doi: 10.1111/j.1464-5491.2006.01845.x

22. Adeline Nyamathi, Benissa E. Salem, Visha Meyer, Kalyan K. Ganguly, Sanjeev Sinha, and Padma Ramakrishnan. 2012. Impact of an Asha Intervention on Depressive Symptoms among Rural Women Living with AIDS in India: Comparison of the Asha-Life and Usual Care Program. AIDS Education and Prevention 24, 3: 280-293. http://doi.org/10.1521/aeap.2012.24.3.280

23. Josefien van Olmen, Grace Marie Ku, Maurits van Pelt, et al. 2013. The effectiveness of text messages support for diabetes self-management: protocol of the TEXT4DSM study in the democratic Republic of Congo, Cambodia and the Philippines. BMC Public Health 13, 1: 423. http://doi.org/10.1186/1471-2458-13-423

24. Neil Patel, Deepti Chittamuru, Anupam Jain, Paresh Dave, and Tapan S. Parikh. 2010. Avaaj Otalo: A Field Study of an Interactive Voice Forum for Small Farmers in Rural India. In Proceedings of the SIGCHI Conference on Human Factors in Computing Systems (CHI '10), 733-742. http://doi.org/10.1145/1753326.1753434

25. Vikram Patel, Somnath Chatterji, Dan Chisholm, et al. 2011. Chronic diseases and injuries in India. The Lancet 377, 9763: 413-428.

26. Trevor Perrier, Nicola Dell, Brian DeRenzi, et al. 2015. Engaging Pregnant Women in Kenya with a Hybrid Computer-Human SMS Communication System. In of the SIGCHI Conference on Human Factors in Computing Systems (CHI '15), 1429-1438, ACM Press. http://doi.org/10.1145/2702123.2702124

27. J. D. Piette. 2000. Interactive voice response systems in the diagnosis and management of chronic disease. The American Journal of Managed Care 6, 7: 817-827.
28. John D. Piette, Milton O. Mendoza-Avelares, Martha Ganser, Muhima Mohamed, Nicolle Marinec, and Sheila Krishnan. 2011. A Preliminary Study of a Cloud-Computing Model for Chronic Illness Self-Care Support in an Underdeveloped Country. American Journal of Preventive Medicine 40, 6: 629-632. http://doi.org/10.1016/j.amepre.2011.02.014

29. Robin Reid Rudy, Lawrence B. Rosenfeld, John P. Galassi, Joanna Parker, and Rachel Schanberg. 2001. Participants' Perceptions of a Peer-Helper, TelephoneBased Social Support Intervention for Melanoma Patients. Health Communication 13, 3: 285-305. http://doi.org/10.1207/S15327027HC1303_4

30. Jahanzeb, Sherwani., Nosheen Ali, Sarwat Mirza, Anjum Fatma, Yousuf Memon, Mehtab Karim, Rahul Tongia, and Roni Rosenfeld. "Healthline: SpeechBased Access to Health Information by Low-Literate users." In Information and Communication Technologies and Development, 2007. ICTD 2007. International Conference on, pp. 1-9. IEEE, 2007.

31. Elizabeth L. Tung and Monica E. Peek. 2015. Linking Community Resources in Diabetes Care: a Role for Technology? Current Diabetes Reports 15, 7: 1-9. http://doi.org/10.1007/s11892-015-0614-5

32. Aditya Vashistha, Edward Cutrell, Gaetano Borriello, and William Thies. 2015. Sangeet Swara: A Community-Moderated Voice Forum in Rural India. In Proceeding of the SIGCHI Conference on Human Factors in Computing Systems (CHI '15), 417-426. http://doi.org/10.1145/2702123.2702191

33. Jerome White, Mayuri Duggirala, Krishna Kummamuru, and Saurabh Srivastava. 2012. Designing a Voice-based Employment Exchange for Rural India. Proceedings of the Fifth International Conference on Information and Communication Technologies and Development, ACM, 367-373. http://doi.org/10.1145/2160673.2160717

34. gramvaani | community-powered-technology. Retrieved September 1, 2015 from http://www.gramvaani.org/

35. Community Radio In India: The Key To Propel Rural Development. Youth Ki Awaaz. Retrieved August 26, 2015 from http://www.youthkiawaaz.com/2011/02/communityradio-for-rural-development/ 